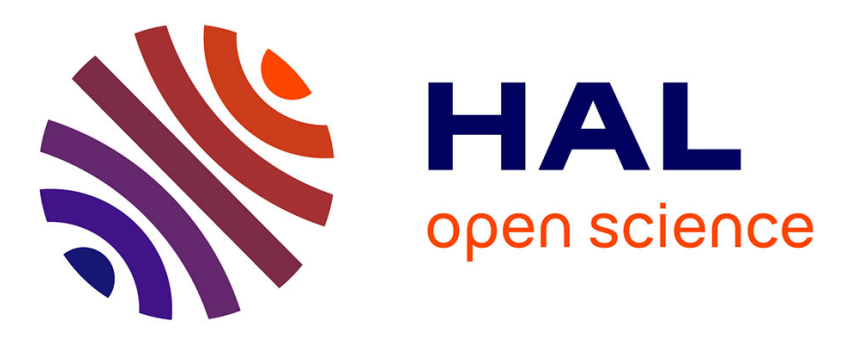

\title{
Space-time evolution of optical breathers and modulation instability patterns in metamaterial waveguides
}

\author{
Gang Xu, Jim Mcniff, Allan Boardman, Bertrand Kibler
}

\section{To cite this version:}

Gang Xu, Jim Mcniff, Allan Boardman, Bertrand Kibler. Space-time evolution of optical breathers and modulation instability patterns in metamaterial waveguides. Wave Motion, 2020, 93, pp.102448. 10.1016/j.wavemoti.2019.102448 . hal-03066389

\section{HAL Id: hal-03066389 \\ https://hal.science/hal-03066389}

Submitted on 15 Dec 2020

HAL is a multi-disciplinary open access archive for the deposit and dissemination of scientific research documents, whether they are published or not. The documents may come from teaching and research institutions in France or abroad, or from public or private research centers.
L'archive ouverte pluridisciplinaire HAL, est destinée au dépôt et à la diffusion de documents scientifiques de niveau recherche, publiés ou non, émanant des établissements d'enseignement et de recherche français ou étrangers, des laboratoires publics ou privés. 


\title{
Space-time evolution of optical breathers and modulation instability patterns in metamaterial waveguides
}

\author{
Gang Xu ${ }^{1}$, Jim McNiff ${ }^{2,3}$, Allan Boardman ${ }^{3, \uparrow}$, and Bertrand Kibler ${ }^{1, *}$ \\ ${ }^{1}$ Laboratoire Interdisciplinaire Carnot de Bourgogne, UMR 6303 CNRS - Université Bourgogne \\ Franche-Comté, 9 avenue Alain Savary, BP 47870, 21078 Dijon Cedex, France \\ ${ }^{2}$ Original Perspectives Ltd, Tewkesbury, United Kingdom \\ ${ }^{3}$ Joule Physics Laboratory, University of Salford, Manchester, United Kington \\ *Corresponding author: \\ *E-mail address: bertrand.kibler@u-bourgogne.fr \\ Tel.: +33380395932
}

\begin{abstract}
We present numerical and theoretical investigations of the spontaneous emergence of noise-driven modulation instability patterns in a metamaterial waveguide, which involves the generation of optical breather waves such as the Peregrine soliton, Akhmediev breathers and Kuznetsov-Ma breathers. We show that the intrinsic properties of the metamaterial waveguide, e.g. self-steepening and the magnetooptics effects, offer the potential to control the formation and subsequent spectral and temporal dynamics of these localized nonlinear waves. Such internal or external perturbations break the symmetry of the spectrum of nonlinear waves, thus leading to the existence of a controllable characteristic group velocity in their space-time evolution.
\end{abstract}

Keywords: Modulation instability, nonlinear optics, breather waves, metamaterials, rogue waves. 


\section{Introduction}

The field of metamaterials has attracted considerable attention during the last two decades due to the exciting potential applications ranging from superlenses, antennas and cloaking devices to sub-wavelength optical waveguides. Optical metamaterials offer the potential to engineer electromagnetic properties in ways that cannot be achieved with materials found in nature, such as the well-known example of negative refractive index, by means of artificially structured materials which exert influence over electromagnetic waves at the sub-wavelength level [1-5]. Nonlinear optics has been also widely impacted by the emergence of the field of electromagnetic metamaterials [6-7]. Nonlinear metamaterials can exhibit some exotic advantages for light manipulation and a rich variety of exciting nonlinear phenomena. In particular, exceptionally strong nonlinearities can be constructed as well as the possibility of efficient dispersion engineering. Among nonlinear phenomena, the formation and propagation of nonlinear guided waves such as temporal and spatial solitons have already been investigated in detail, particularly by considering integrated waveguide configurations based on a negative-index metamaterial (i.e., with both negative dielectric permittivity and negative magnetic permeability) with a Kerr-type nonlinearity [7-11]. In a similar manner, the standard linear stability analysis of the homogeneous steady state has been performed in various propagation schemes to predict the new regimes of modulation instability [12-15]. In general, investigating modulation instability (MI) and short pulse propagation in a nonlinear double negative metamaterial gives rise to an extended nonlinear Schrödinger equation (NLSE) modeling, where higher-order dispersion and nonlinearity strongly impact the space-time trajectories of localized nonlinear waves, including optical solitons and optical rogue waves [16].

In this paper, we extend previous studies to the spontaneous emergence of strongly localized nonlinear waves through modulation instability in a negative-index metamaterial waveguide. In particular, we numerically investigate noise-induced modulation instability patterns formed by breather waves (i.e., unstable pulsating solutions of the NLSE [17]). We evidence that such breather waves are influenced, but not suppressed, in a nonlinear metamaterial system. More specifically, we focus our discussion on the impact of self-steepening effect on both the spectral and temporal dynamics of the modulation instability patterns. We demonstrate that, in the temporal domain, the MI patterns propagate with a characteristic group velocity, which 
corresponds to typical asymmetric spectra. Finally, by taking advantage of possible introduction of the magnetooptic property in the metamaterial waveguide, we provide important control capabilities over temporal behaviors by carefully adjusting the external magnetic field.

\section{Breather waves and modulation instability}

A typical waveguide structure used here is a planar structure with a metamaterial core that has boundaries in $\mathrm{x}$ and $\mathrm{y}$ directions and propagation is along the z-axis (see also section 6 of Ref. [16]). A part of the structure, in the form of the substrate, is replaced with a magnetooptic material. Here the magnetic field is applied in what is known as a Voigt configuration. This has an externally applied magnetic field in the plane of the guide perpendicular to the direction of optical propagation, which here can be applied along either the positive or negative $\mathrm{x}$-direction. We also assume that the core material is isotropic and it has a negative permittivity and negative permeability thus the form of the metamaterials considered here is transparently double-negative [11]. The use of double negative media is the way forward where appropriate extensions to the NLSE have already been developed [6,14]. Here it is assumed that the nonlinear behaviour discussed originates from an isotropic Kerr dielectric, such that an appropriate extension to the focusing NLSE is utilised that takes into account the metamaterial properties with selfsteepening and magnetooptic effects (see section 6 of Ref. [16] for more details). The form of the extended NLSE is given below in Eq. (1). Note that there is also the capability of adding higher order and Raman scattering effects, however, in the simulations discussed below, these parameters are not involved, but later discussed in the last section.

$$
i \frac{\partial \psi}{\partial \xi}+\frac{1}{2} \frac{\partial^{2} \psi}{\partial \tau^{2}}+\left(\psi+i S \frac{\partial \psi}{\partial \tau}\right)|\psi|^{2}+v \psi=0 .
$$

Here $\psi$ is an electromagnetic wave envelope which is a function of $\xi$ (a scaled propagation

distance) and $\tau$ (a co-moving time with the wave-group velocity). $S$ is the self-steepening coefficient or shock term, and $v$ stands for the magnetooptic effect.

First, we recall some of the features of modulation instability whose essential space-time dynamics can be well described by breather solutions in the standard NLSE framework [17-19], 
when $S=v=0$ in Eq. (1). First-order breather solutions, also known as solitons on finite background, can be simply written in the following form [20]:

$$
\psi(\xi, \tau)=e^{i \xi}\left[1+\frac{2(1-2 a) \cosh (b \xi)+i b \sin (b \xi)}{\sqrt{2 a} \cos (\omega t)-\cosh (b \xi)}\right] .
$$

The physical behavior of this solution is governed by a single parameter $a$ through function arguments $b=\sqrt{8 a(1-2 a)}$ and $\omega=2 \sqrt{1-2 a} . \omega$ and $b$ have physical significance as the modulation frequency and exponential growth and decay rate. The space-time evolution of resulting localized wave structures strongly depends on the parameter $a$. For $0<a<0.5$, Eq. (2) describes the Akhmediev breather (AB) corresponding to a single growth and decay cycle of the initial weak time-periodic modulation of the plane. For $a=0.5$, the solution then describes the Peregrine breather (PB) corresponding to the low frequency limit of the $\mathrm{AB}$ and characterized by the localization in both temporal and longitudinal dimensions. While for $a>$ 0.5 , the solution describes the Kuznetsov-Ma breather (KMB), hyperbolic trigonometric functions in Eq. (2) become ordinary circular functions and vice-versa, thus the breather solution is now periodic in space and localized in time. Note that from Eq. (2) the Peregrine breather solution, or rogue-wave solution, can also be simplified into the following particular fractional form:

$$
\psi(\xi, \tau)=\left[1-\frac{4(1+2 i \xi)}{1+4 \tau^{2}+4 \xi^{2}}\right] e^{i \xi}
$$

We performed NLSE simulations with a noise-perturbed plane wave initial condition (see similar analysis in Ref. [19]) to show the noise-driven emergence of breather waves (i.e., nonlinear MI dynamics) in space and time. Figure 1 depicts the resulting temporal and spectral evolutions of the MI patterns spontaneously generated. In the time domain, see Fig. 1(a), we can firstly identify the breaking of the initial plane wave into a quasi-periodic pulse train around $\xi \sim 13$ that subsequently disappear. Further propagation indicates several growth and decay cycles of localized wave structures with more chaotic evolution, which involves their mutual interactions. Overall, these MI patterns originating from noise exhibit clear signatures of the breather solutions described above. For example, the first temporal peaks emerging at $\xi \sim 12.4$ are found for $70<\tau<90$ (see white dashed rectangle in the right panel), they exhibit a temporal period $\Delta \tau \sim 4.4$, which corresponds to the MI frequency of maximum gain (i.e., $\omega=$ $\frac{2 \pi}{\Delta \tau} \sim \sqrt{2}$ ), the exponential growth of this frequency is clearly observed in the spectral domain at 
$\xi \sim 10$ (see Fig. 1(b)). A cascade of MI gain bands is observed with further propagation; this spectral broadening is related to the emerging localized temporal structures. Their quasi-timeperiodic intensity profile can be well-fitted by using $A B$ solution with governing parameter calculated from MI frequency of maximum gain (i.e., $a=0.25$ ), as shown in Fig. 2(b) without any adjusting parameter. Note that another series of $\mathrm{AB}$ appear, e.g., at $\xi \sim 12.9$, as indicated in the left panel of Fig. 1(a) and in Fig.2 (a), whose corresponding governing parameter is $a=0.2$.
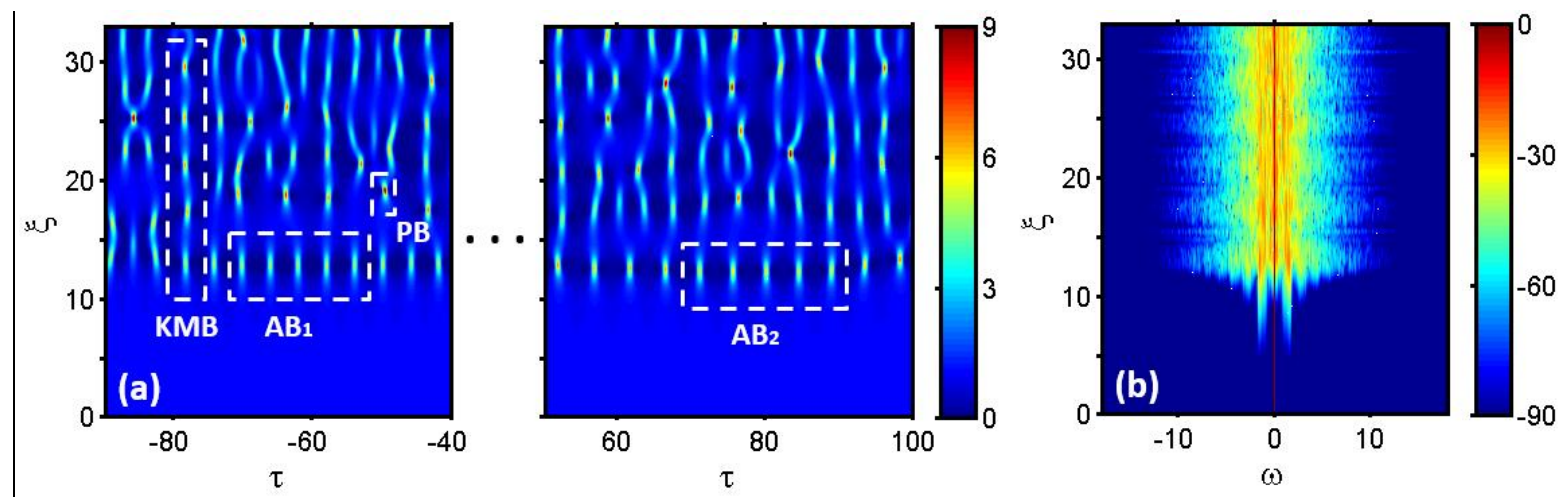

Figure 1: (a) Density map showing the space-time evolution of noise-driven modulation instability of the plane wave in the NLSE framework. Left and right panels presents the detailed dynamics obtained in two regions of the full temporal window simulated, namely for (left) $-90<\tau<-40$ and (right) $50<\tau<100$. White dashed rectangles emphasize typical MI patterns related to AB, KMB and PB solutions of the NLSE. (b) Density map of the corresponding evolution of the power spectrum.

By analyzing the full evolution map from Fig. 1(a), we can clearly identify other typical MI patterns fitted by ideal breather solutions, namely KMB and PB. In Fig.2 (c), we report the space-periodic profile of the KMB-like structure found near $\tau \sim-75$ in Fig.1(a). Its evolution along $\xi$-axis agrees with the analytical KMB solution based on $a=0.7$. Finally, a doubly localized wave structure, located at $\tau \sim-48$ and $\xi \sim 18$ in Fig.1 (a), is found to be nearly identified as a PB (see comparison in Fig. 2(d)). Note that higher-order breather structures can also be generated through collision processes in these noise-induced MI patterns. It is also worth mentioning that after the first spectral broadening related to initial $\mathrm{AB}$ emergence, the power spectrum is characterized by a universal triangular shape in logarithmic scale (typical feature of 
breather waves, see Ref. [18-22]) whose bandwidth oscillates in a chaotic fashion related to space-time interactions between localized wave structures.

In the following, we will make use of the same selected random noise superimposed to the initial plane wave for all the numerical simulations. We will introduce both higher-order nonlinearity and magnetooptic effect to investigate their respective influence on the temporal and spectral dynamics of MI patterns.
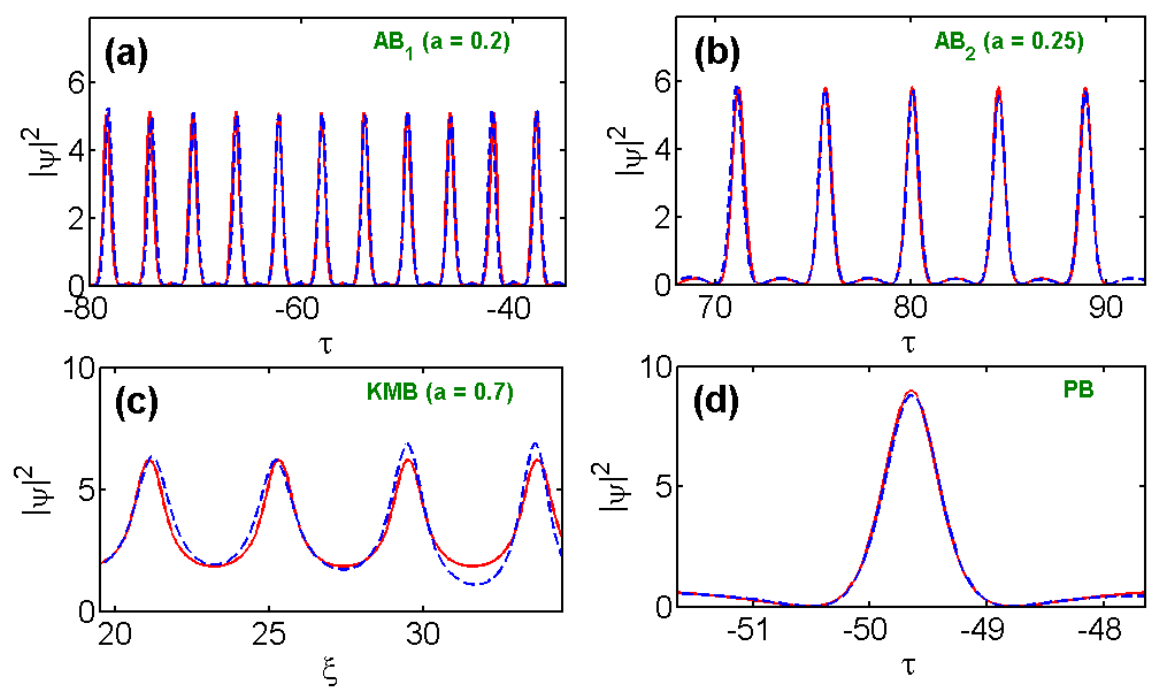

Figure 2: The dashed-blue line in (a-d) show the intensity profiles extracted from the region of chaotic MI field indicated in Fig. 1(a) for $\mathrm{AB}$ (two different series, one at $-80<\tau<$ $-30, \xi=12.9$ and another one at $70<\tau<90, \xi=12.4)$ KMB $(20<\xi<35, \tau=79)$ and PB (localized at $\xi=19, \tau=-49.7$ ) respectively. These profiles are compared with corresponding breather solutions of the NLSE (red solid line).

\section{Impact of self-steepening term}

In the last section, we presented the temporal and spectral evolutions of the noise-induced MI patterns, whose nonlinear dynamics can be described by the standard NLSE and its breather solutions. However, when the time duration of these localized patterns are short enough (i.e., their spectrum large enough), the dependence of the group velocity upon the intensity of the pulse will cause a pulse to change shape. In a normal positive phase medium, the trailing edge of the pulse will steepen, whereas in a metamaterial this can result in the steepening of the leading 
edge of the pulse [11]. In this case, the description of the dynamics requires an extended version of NLSE (see Eq. 1) including the self-steepening term (also known as the dispersion of the nonlinearity) that can be negative or positive. Self-steepening leads to an asymmetry in the spectral broadening processes of ultrashort pulses [23]. More particularly, the self-steepening coefficient involves very specific properties of the metamaterial and it is characterized by a strong frequency dependence which significantly impacts soliton dynamics. In this section, we will discuss the impact of self-steepening term which introduces some interesting features of the temporal-spectral dynamics of the MI patterns. We selected a self-steepening coefficient $S$ in line with that of previous studies [11, 16, 24-27]. As already known, a slight deviation from the ideal NLSE model will induce a deviation from the expected theoretical solutions and their space-time dynamics [16].
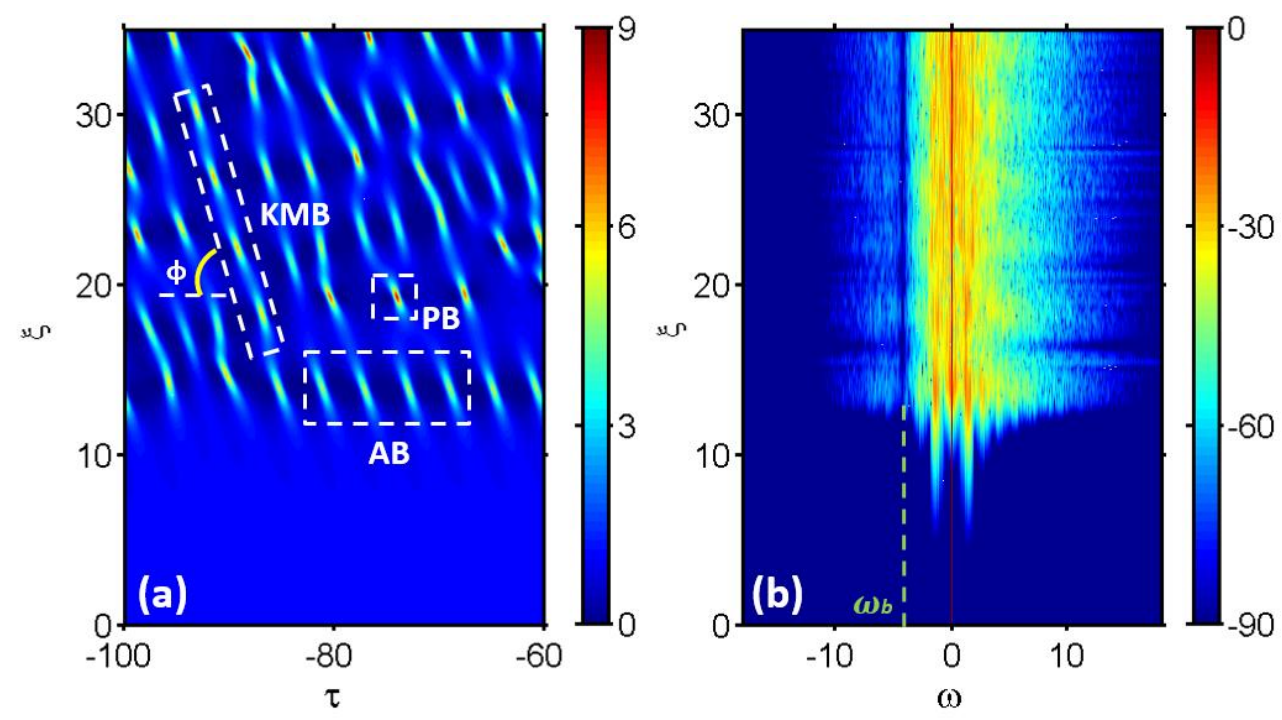

Figure 3: (a) Density map showing the temporal (a) and spectral (b) evolution of noisedriven modulation instability of the plane wave in the extended NLSE framework including the self-steepening term ( $S=-0.24)$.

Taking the same initial condition of the noise-perturbed plane wave as in the Fig. 1, we performed the numerical simulation involving the self-steepening term with $S=-0.24$. Figure 3 (a-b) illustrates the temporal and spectral evolutions of the MI patterns and there are several key observations: 
(i) In the temporal domain (see Fig. 3a), the MI patterns propagate with a characteristic group velocity $v_{g}$ which differs from the group velocity of the carrier wave. All the breathing structures then experience a "homogeneous shift" toward the negative direction with respect to the time axis. All the peaks of localized structures have similar peak powers to those in Fig. 1, but they are now aligned in a specific orientation and rotated counter clockwise. A direct piece of evidence is that the example of KMB-like trajectory remains linear in the space-time diagram (see white rectangle in Fig. 3a). Because of the non-integrability of the extended NLSE, rigorous analytical expression of $v_{g}$ for the KMB-like structure is a challenging task. However, one can easily estimate the change in group velocity from the deviation of KMB pattern: $\Delta v_{g}=$ $\cot \phi=\frac{d \tau}{d \xi} \sim-0.49$. This kind of tilted breather structures was also observed in the case of the integrable Sasa-Satsuma or Hirota equation [24-25]. The resulting nonlinear wave then makes an angle with the propagation axis, which corresponds here to an effective group velocity proportional to the self-steepening coefficient. Simply by changing the sign of $S$, we can observe the opposite time-shift of the overall MI pattern. To understand the relation $\Delta v_{g}$ and $S$, we can recall that the Kerr effect leads to a change in the phase velocity of a pulse, whereas the selfsteepening alters the group-velocity of the pulse twice as strongly as the nonlinear phase shift alters the phase velocity (simply due to the time-derivative term in the extended NLSE) [23]. As we are here considering quasi-continuous waves, the impact of self-steepening on the group velocity can be then approximated by a constant value over time, which is twice the selfsteepening coefficient. This explains why the MI patterns experience this "homogeneous shift" with respect to the time axis, which can be seen as a cross-phase modulation effect induced by the continuous background.

(ii) In the frequency domain (see Fig. 3b), the self-steepening terms induces a strong asymmetry of the power spectrum with respect to the carrier wave frequency as soon as the localized ultrashort structures are generated $(\xi>12)$. A long-tailed spectrum can be noticed in the positive frequency region. Such strong spectral asymmetries were already revealed in the framework of the integrable Sasa-Satsuma equation [26]. By using the dispersion relation, we can calculate the weight-average frequency of the power spectrum, thus giving another estimation of the evolution of group velocity [27-28]:

$$
v_{g}(\xi) \approx \beta_{2}\left[\overline{\omega(\xi)}-\omega_{0}\right]
$$


In this expression, $\omega_{0}$ is the angular frequency of the carrier wave, while

$$
\overline{\omega(\xi)}=\frac{\int|\widetilde{\psi}(\omega, \xi)|^{2} \omega d \omega}{\int|\widetilde{\psi}(\omega, \xi)|^{2} d \omega}
$$

is the weight-average angular frequency and $|\tilde{\psi}|^{2}$ is the power spectrum (note that $\tilde{\psi}(\omega, \xi)=$ $\left.\int \psi(\xi, \tau) e^{-i \omega \tau} d \tau\right)$. For a symmetric MI spectrum, the value of $\Delta v_{g}$ in the co-moving frame is null, which is confirmed by the numerical simulation without the presence of the self-steepening term (see Fig. 1). When calculating Eqs. (5-6) with fully developed MI spectra reported in Fig. 3(b), we find the evolution of $\Delta v_{g}$ shown in Fig. 4. There are three regimes: (1) for $0<\xi<6$, the optical wave almost remains a plane wave for which the impact of the self-steepening term can be neglected. That is why in this stage, the initial spectrum is still symmetric; (2) for $6<$ $\xi<13$, significant spectral broadening occurs related to the exponential growth of localized breather structures so that the self-steepening term plays an important role, thus resulting in the asymmetry of the spectrum and the strong variation of group velocity; (3) for $\xi>13$, the spectral broadening saturates as well as the change of group velocity. In Fig. 4, we also compare the evolution of group velocity with the estimated value $\Delta v_{g}$ from the KMB pattern in Fig. 3a. We obtained a good agreement and the discrepancies result from the fact that the first approach only considers one breathing structure whereas the latter include the overall temporal profile. This also explains the noticeable oscillations of $\Delta v_{g}$ sensitive to transient space-time interactions.

(iii) Besides the overall asymmetry of MI spectra, we also reveal that a zero-intensity frequency is present in fully-developed MI spectra (similarly to Ref. [26]). This phenomenon can be simply related here to the fact that our extended NLSE (Eq. 1) still conserves the "number of photons" [29]:

$$
N=\int \frac{|\widetilde{\psi}(\omega, \xi)|^{2}}{1-S \omega} d \omega=c s t
$$

Indeed we can predict the existence of a forbidden angular frequency $\omega_{b}=1 / S$ since the denominator of eq. (7) should not be null. The calculated frequency $\omega_{b}=-4.167$ fits well the positon of the spectral dip (dark blue) observed in our simulations (indicated by the dashed green line in Fig. 3b). 


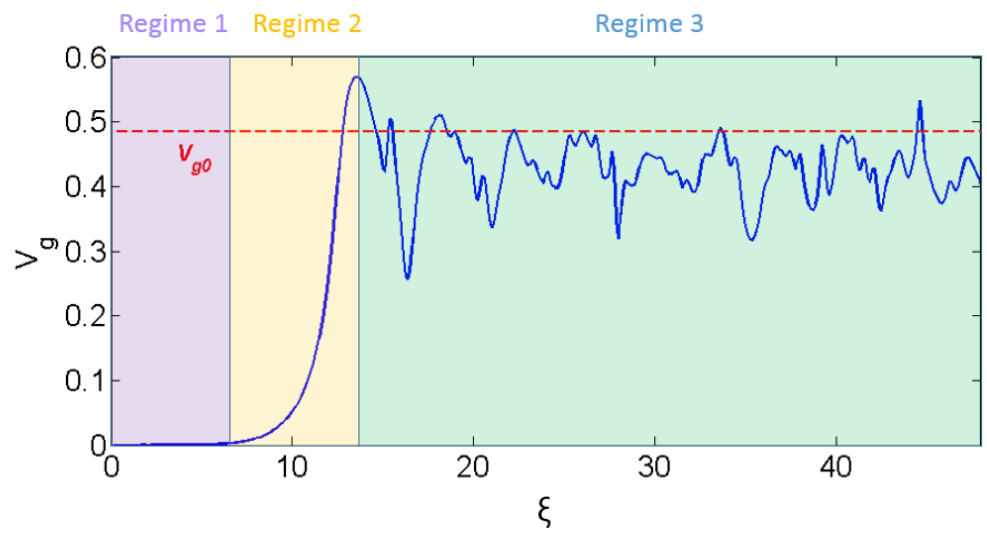

Figure 4: Evolution of calculated $v_{g}$ as a function of $\xi$. Dashed-red line corresponds to the characteristic group velocity from space-time KMB pattern. Three distinct regimes are denoted and described in the text.

The above simulations show that localized structures in the form of NLSE breathers still persist in MI pattern when the self-steepening term is present. In Fig. 5, we underline that the impact of self-steepening on MI pattern and breathers mainly consists in a change of their group velocity. By performing the following change of variables: $\tau^{\prime}=\tau_{0}+\xi v_{g 0}, \xi^{\prime}=\xi$ for the analytical expression (2) of $\mathrm{KMB}$ and $\mathrm{AB}$, we are able to fully describe the intensity profiles of characteristic patterns selected in Fig. 3(a). Note that the application of Galilean transformation on Akhmediev breathers was also demonstrated in Ref. [30].
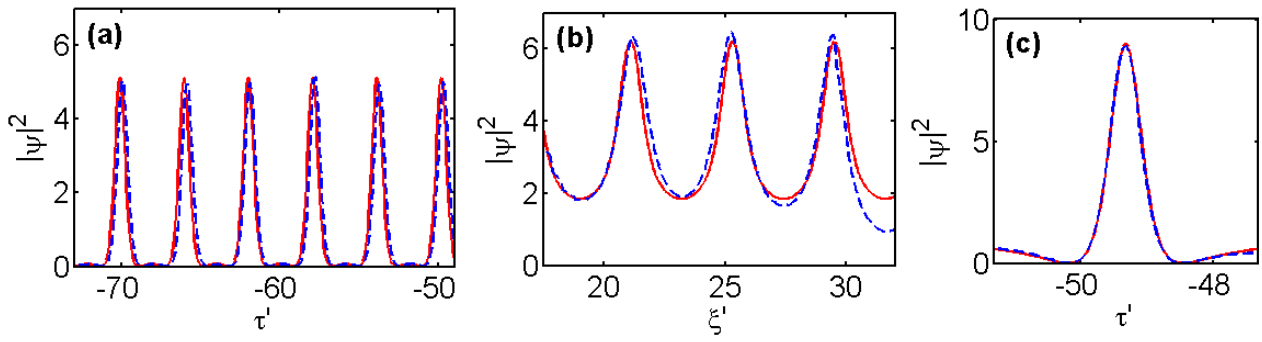

Figure 5: Recovery of the intensity profile of $\mathrm{AB}$ (a), KM (b) and PS (c) with the Galilean transformation $\tau^{\prime}=\tau_{0}+\xi v_{g 0}, \xi^{\prime}=\xi$. Solid red lines are the analytical solutions; Dashed blue lines are the numerical simulations. 


\section{Impact of magnetooptic effect}

In this section, we now focus on the impact of the magnetooptic effect on the evolution of the MI patterns. The contribution of magnetooptic effect is included in the extended NLSE (Eq. 1), where the expression of $v$ is written as [16]:

$$
v\left(\tau+\frac{\xi}{v_{m}}\right)=\frac{v_{\max }}{2}\left[1-\tanh \left(\frac{\tau+\left(\frac{\xi}{v_{m}}\right)-T_{v}}{\Delta T_{v}}\right)\right]
$$

where $T_{v}$ is the delay of the magnetization after excitation by the electromagnetic input, and $\Delta T_{v}$ is the normalized time over which the magnetization takes place, while $v_{m}$ is the group velocity and $v_{\max }$ is the maximum value of the magnetization. Taking the same initial condition of the noise-perturbed plane wave as in the Fig. 1, we first perform the numerical simulation including the magnetooptic effect in Eq. (1) without the self-steepening term (i.e., with $S=0$ ). Noteworthy, according to Eq. (8), the presence of the magnetooptic influence is $\xi$-dependent because of the appearance of longitudinal parameter $\xi$ in the function of $v$. It means that for the current problem of precise wave manipulation in the metamaterial, it is possible to switch-on and switch-off the contribution of the magnetooptic effect, so that it can be accurately synchronized with the emergence of $\mathrm{AB}, \mathrm{KMB}$ and $\mathrm{PB}$ from the initial noise. For example, with parameter chosen here $\left(v_{\max }=-17, T_{v}=200, v_{m}=0.03, \Delta T_{v}=10\right)$, the magnetooptic effect begins to play a role at $\xi \sim\left(-\tau_{0}+T_{v}\right) v_{m}=6$, where $\tau_{0}$ is the ending point of the time window (to simplify, here we have $\tau_{0}=0$ ). As shown in Fig. 6, at this location, in the temporal domain, AB, KMB and $\mathrm{PB}$ are to be excited, while in the spectral domain, at $\xi \sim 6$, a slight discontinuity can be noticed. Here, the presence of the magnetooptic effect also leads to the generation of a small asymmetry in the spectrum and a characteristic change of group velocity of MI patterns in the time domain. In fact, this characteristic velocity depends on the direction and the amplitude of the external magnetic field. For the parameters used, the change in the average group velocity is opposite to the one induced by a negative self-steepening coefficient. There is an overall clockwise rotation of the MI pattern without cancelling the main features of breather structures. Now it becomes obvious to investigate the possibility to counteract the deformations of localized wave structures induced by the self-steepening term, by properly adding an external magnetic field over the metamaterial. 

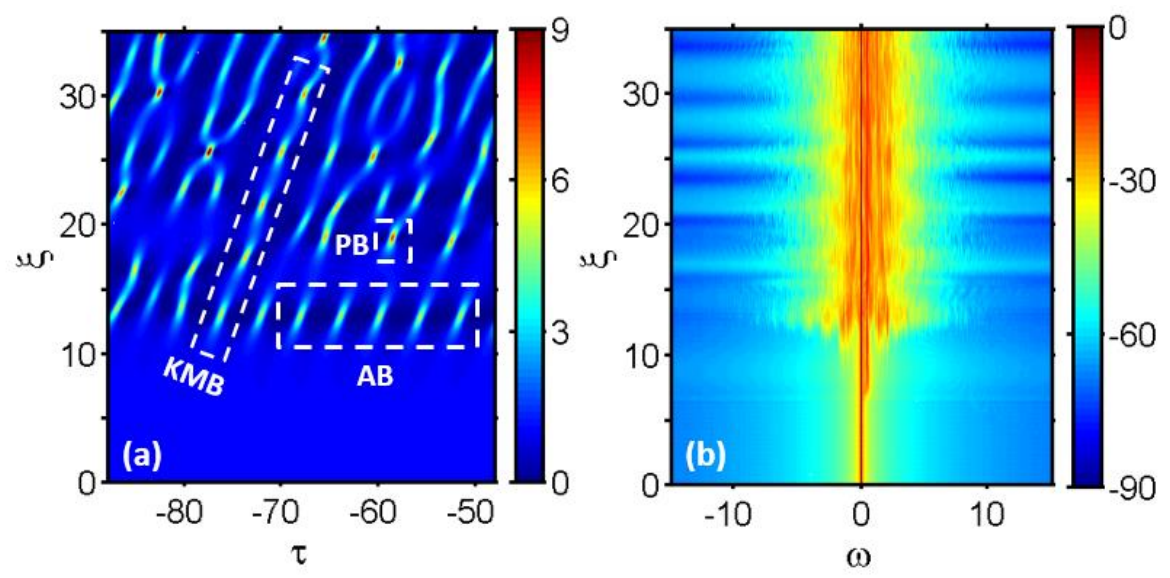

Figure 6: Density map showing the temporal (left panel) and spectral (right panel) evolution of noise-driven modulation instability of the plane wave in the extended NLSE framework including the magnetooptic effect $\left(v_{\max }=-17\right)$.

Next we perform the numerical simulation implementing both contributions of the selfsteepening term and the magnetooptic effects. As shown in Fig. 7, the impact of self-steepening effect and the magnetooptic effect can cancel each other, which means that the change of the characteristic group velocity turns to be nearly zero. However, it is worth mentioning that the fully-developed MI spectrum remains asymmetric with only some of the typical features previously described, mainly the tailed spectrum induced by self-steepening term in the positive frequency region. When looking at the intensity profiles of the characteristic breather-like waves, we can again fit them with the standard breather solutions without any transformation.

Finally, we performed a series of simulations of the complete form of Eq. (1) in order to find the value of magnetic field required to minimize the effect of distinct values of self-steepening coefficient on MI patterns. Figure 8 provides the corresponding couples of values obtained for the magneticoptic coefficient $v$ and the self-steepening coefficient $S$. In particular, we find a linear relation between these two parameters which can be approximately expressed as $v \approx$ $66.7 S$. 

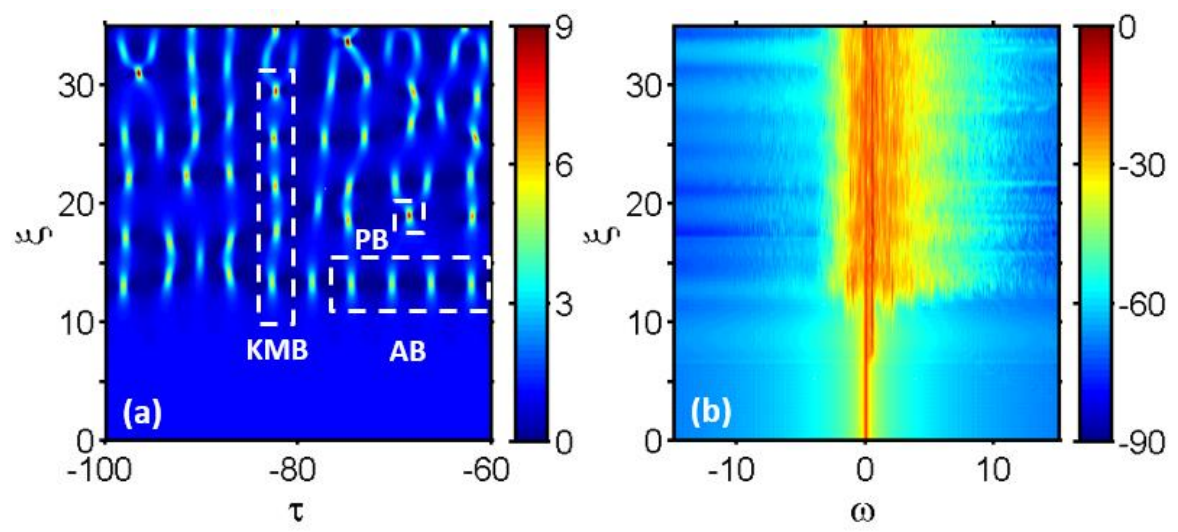

Figure 7: Density map showing the temporal (left panel) and spectral (right panel) evolution of noise-driven modulation instability of the plane wave in the extended NLSE framework including both the self-steepening $(S=-0.24)$ and the magnetooptic $\left(v_{\max }=-17\right)$ effects.

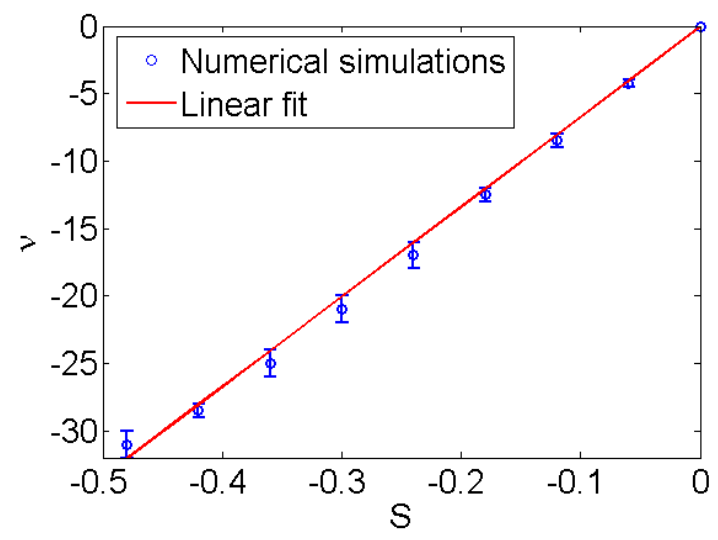

Figure 8: Dependence of the magnetooptic coefficient $v$ on the self-steepening coefficient $S$ to cancel the impact of each other. Results obtained from numerical simulations of the complete form of Eq. (1) are blue circles with error bars (the red-solid line shows the corresponding linear fit).

\section{Discussion and conclusion}

To conclude, we investigated the spontaneous generation of MI patterns in a transparently double-negative metamaterial waveguide modeled by an appropriate extension of the NLSE. Typical breather dynamics are clearly observed and almost identical to $\mathrm{AB}, \mathrm{KMB}$, and $\mathrm{PB}$ solutions of the NLSE. The intrinsic properties of the metamaterial waveguide, e.g. selfsteepening and the magnetooptics effects, offer the potential to control the formation and subsequent spectral and temporal dynamics of these localized nonlinear waves. The presence of 
the self-steepening effect induces a characteristic group velocity in the space-time evolution of MI patterns. Via the magnetooptic property, the impact of the self-steepening term can be cancelled by judiciously adding an external magnetic field. Our relevant numerical simulations reveal that there is a simple linear relation between the magnetooptic coefficient and the selfsteepening coefficient to compensate for the impact of each other. These results can be applied to the search for precise space-time trajectories of nonlinear waves in integrated waveguides. In general, all these extensions or perturbations of the NLSE then break the symmetry of the spectrum of breather waves, but without preventing their emergence or propagation, and we reveal particular signatures of the presence of self-steepening and magnetooptic perturbations.

To go beyond the present approach, in the framework of nonlinear dynamics governed by the generalized NLSE [23], higher order nonlinear effects, such as the spontaneous Raman scattering and higher order dispersion terms, may also break the spectrum symmetry, and induce more complex trajectories of the MI patterns in the time domain. These higher order effects will add a constant or even variable accelerations on the MI patterns, which means that their trajectories will be more or less "out of control" when simply applying the external magnetic field. In this case, in order to systematically manipulate their spectro-temporal dynamics, more advanced technics and investigations are a very challenging subject of future studies. To this end, it is worth mentioning recent theoretical efforts made to extend the existence of breather and rogue wave solutions towards more complex extensions of the NLSE [31-34].

\section{Acknowledgements}

This research was supported by the French "Investissement d'Avenir" program (PIA2/ISITE BFC, contract ANR-15-IDEX-03). The authors are grateful to A. Picozzi for the stimulating discussion. This paper is dedicated to the memory of Professor Allan Boardman who was unfortunately not around to see its completion but played an inspirational role in the study of electromagnetic rogue waves in metmaterials and many other works. His input will be greatly missed.

\footnotetext{
$\dagger$ Deceased
} 


\section{References}

[1] N. Engheta and R. W. Ziolkowski, Metamaterials: Physics and Engineering Explorations. John Wiley \& Sons, 2006.

[2] L. Solymar and E. Shamonina, Waves in Metamaterials. Oxford University Press, Oxford, 2009.

[3] W. Cai and V. Shalaev, Optical Metamaterials: Fundamentals and Applications. SpringerVerlag, New York, 2010.

[4] S. Zhang, W. Fan, N. C. Panoiu, K. J. Malloy, R. M. Osgood, and S. R. J. Brueck, 'Experimental Demonstration of Near-Infrared Negative-Index Metamaterials', Phys. Rev. Lett., vol. 95, no. 13, p. 137404 (2005).

[5] S. Xiao et al., 'Loss-free and active optical negative-index metamaterials', Nature 466, 735738 (2010).

[6] M. Scalora et al., 'Generalized nonlinear Schrödinger equation for dispersive susceptibility and permeability: application to negative index materials', Phys. Rev. Lett. 95, 013902 (2005).

[7] M. Lapine, I. V. Shadrivov, and Y. S. Kivshar, 'Colloquium: Nonlinear metamaterials', Rev. Mod. Phys. 86, 1093-1123 (2014).

[8] I. V. Shadrivov, A. A. Sukhorukov, Y. S. Kivshar, A. A. Zharov, A. D. Boardman, and P. Egan, 'Nonlinear surface waves in left-handed materials', Phys. Rev. E 69, 016617 (2004).

[9] A. D. Boardman, P. Egan, L. Velasco, and N. King, 'Control of planar nonlinear guided waves and spatial solitons with a left-handed medium', J. Opt. A 7, S57 (2005).

[10] A. D. Boardman, R. C. Mitchell-Thomas, N. J. King, and Y. G. Rapoport, 'Bright spatial solitons in controlled negative phase metamaterials', Opt. Commun. 283, 1585-1597 (2010).

[11] A. D. Boardman, O. Hess, R. C. Mitchell-Thomas, Y. G. Rapoport, and L. Velasco, 'Temporal solitons in magnetooptic and metamaterials waveguides', Photon. Nanostruct.Fundam. Appl. 8, 228-243 (2010).

[12] I. Kourakis and P. K. Shukla, 'Nonlinear propagation of electromagnetic waves in negativerefraction-index composite materials', Phys. Rev. E 72, 016626 (2005).

[13] P. Kockaert, P. Tassin, G. Van der Sande, I. Veretennicoff, and M. Tlidi, 'Negative diffraction pattern dynamics in nonlinear cavities with left-handed materials', Phys. Rev. A 74, 033822 (2006).

[14] S. Wen, Y. Wang, W. Su, Y. Xiang, X. Fu, and D. Fan, 'Modulation instability in nonlinear negative-index material', Phys. Rev. E 73, 036617 (2006).

[15] Y. Xiang, S. Wen, X. Dai, Z. Tang, W. Su, and D. Fan, 'Modulation instability induced by nonlinear dispersion in nonlinear metamaterials', J. Opt. Soc. Am. B 24, 3058-3063 (2007).

[16] A. D. Boardman et al., 'Waves in hyperbolic and double negative metamaterials including rogues and solitons', Nanotechnology 28, 444001 (2017).

[17] N. Akhmediev and A. Ankiewicz, Solitons, Nonlinear Pulses and Beams. Chapman and Hall, London, 1997.

[18] J. M. Dudley, G. Genty, F. Dias, B. Kibler, and N. Akhmediev, 'Modulation instability, Akhmediev Breathers and continuous wave supercontinuum generation', Opt. Express 17, 21497-21508 (2009).

[19] S. Toenger et al., 'Emergent rogue wave structures and statistics in spontaneous modulation instability’, Sci. Rep. 5, 10380 (2015). 
[20] B. Kibler et al., 'Observation of Kuznetsov-Ma soliton dynamics in optical fibre', Sci. Rep. 2, 463 (2012).

[21] K. Hammani, B. Wetzel, B. Kibler, J. Fatome, C. Finot, G. Millot, N. Akhmediev, and J. M. Dudley, 'Spectral dynamics of modulation instability described using Akhmediev breather theory', Opt. Lett. 36, 2140-2142 (2011).

[22] N. Akhmediev, A. Ankiewicz, J. M. Soto-Crespo, and J. M. Dudley, 'Universal triangular spectra in parametrically-driven systems', Phys. Lett. A 375, 775-779 (2011).

[23] G. P. Agrawal, Nonlinear Fiber Optics, ${ }^{\text {th }}$ ed. Academic Press, Oxford, 2013.

[24] A. Ankiewicz, J. M. Soto-Crespo, and N. Akhmediev, 'Rogue waves and rational solutions of the Hirota equation', Phys. Rev. E 81, 046602 (2010).

[25] A. Ankiewicz, J. M. Soto-Crespo, M. A. Chowdhury, and N. Akhmediev, 'Rogue waves in optical fibers in presence of third-order dispersion, self-steepening, and self-frequency shift', J. Opt. Soc. Am. B 30, 87-94 (2013).

[26] N. Akhmediev, J. M. Soto-Crespo, N. Devine, and N. P. Hoffmann, 'Rogue wave spectra of the Sasa-Satsuma equation', Physica D 294, 37-42 (2015).

[27] J. M. Soto-Crespo, N. Devine, N. P. Hoffmann, and N. Akhmediev, 'Rogue waves of the Sasa-Satsuma equation in a chaotic wave field', Phys. Rev. E 90, 032902 (2014).

[28] G. Xu, A. Gelash, A. Chabchoub, V. Zakharov, and B. Kibler, 'Breather wave molecules', Phys. Rev. Lett. 122, 084101 (2018).

[29] K. J. Blow and D. Wood, 'Theoretical description of transient stimulated Raman scattering in optical fibers', IEEE J. Quantum Electron. 25, 2665 (1989).

[30] N. Akhmediev, J. M. Soto-Crespo, and A. Ankiewicz, 'How to excite a rogue wave', Phys. Rev. A 80, 043818 (2009).

[31] A. Chowdury, D. J. Kedziora, A. Ankiewicz, and N. Akhmediev, 'Breather solutions of the integrable quintic nonlinear Schrödinger equation and their interactions', Phys. Rev. E 91, 022919 (2015).

[32] A. Ankiewicz and N. Akhmediev, 'Rogue wave solutions for the infinite integrable nonlinear Schrödinger equation hierarchy', Phys. Rev. E 96, 012219 (2017).

[33] A. Ankiewicz, M. Bokaeeyan, and N. Akhmediev, 'Rogue waves under influence of Raman delay', J. Opt. Soc. Am. B 35, 899-908 (2018).

[34] S. Chen, F. Baronio, J. M. Soto-Crespo, P. Grelu, and D. Mihalache, 'Versatile rogue waves in scalar, vector, and multidimensional nonlinear systems', J. Phys. A: Math. Theor. 50, 463001 (2017). 